“C2020 IEEE. Personal use of this material is permitted. Permission from IEEE must be obtained for all other uses, in any current or future media, including reprinting/republishing this material for advertising or promotional purposes, creating new collective works, for resale or redistribution to servers or lists, or reuse of any copyrighted component of this work in other works." 


\section{Numerical Study on Electromagnetic Field and AC loss of HTS Air-core Transformer}

\author{
Runtao Zhang, Jianxun Jin \\ School of Electrical and Information \\ Engineering \\ Tianjin university \\ Tianjin, China \\ rtzhang@tju.edu.cn
}

\author{
Jianguo Zhu, Youguang Guo \\ School of Electrical and Data \\ Engineering \\ University of Technology Sydney \\ Sydney, Australia
}

\author{
Ahmed Abu-Siada \\ Electrical and Computer Engineering \\ Discipline \\ Curtin University \\ Perth, Australia
}

\begin{abstract}
Air-core transformer has advantages of no iron loss, no magnetic saturation, and compact in size, which make them attractive to be studied for practical applications. In this paper, the $\boldsymbol{H}$-formulation is used to numerically analyze the electromagnetic field and AC loss of the HTS air-core transformer and optimize the air gap.
\end{abstract}

Keywords-high temperature superconductor (HTS); HTS aircore transfromer; AC loss; electromagnetic field; H-formulation

\section{INTRODUCTION}

With the development of HTS technology, research involving the application of HTS transformers have been actively carried out. Compared with traditional transformers, the main advantages of HTS air-core transformer include no iron loss, no magnetic saturation, a possible reduction of size and weight [1].

This paper briefly introduces the modeling method of HTS air-core transformer and analyze the electromagnetic field distribution, AC loss and efficiency optimization.

\section{NUMERICAL METHOD}

\section{A. Basic Equations}

The primary and secondary sides of the air-core transformer are composed of 8 double pancakes as shown in Fig. 1(a). The PDE module based on the $\boldsymbol{H}$-formulation in the Comsol is established to numerically study and the governing equations are as follows [2]:

$$
\begin{gathered}
\frac{\partial\left(\mu_{0} \mu_{r} H\right)}{\partial t}+\nabla \times(\rho \nabla \times H)=0 \\
\frac{\partial H_{r}}{\partial z}-\frac{\partial H_{z}}{\partial r}=J_{\varphi} \\
E_{\varphi}=\rho J_{\varphi} \\
J_{\varphi}=J_{c}(B)=J_{c 0} /\left(1+\sqrt{\beta^{2}\left|B_{\square}\right|^{2}+\left|B_{\perp}\right|^{2}} / B_{0}\right)^{\alpha}
\end{gathered}
$$

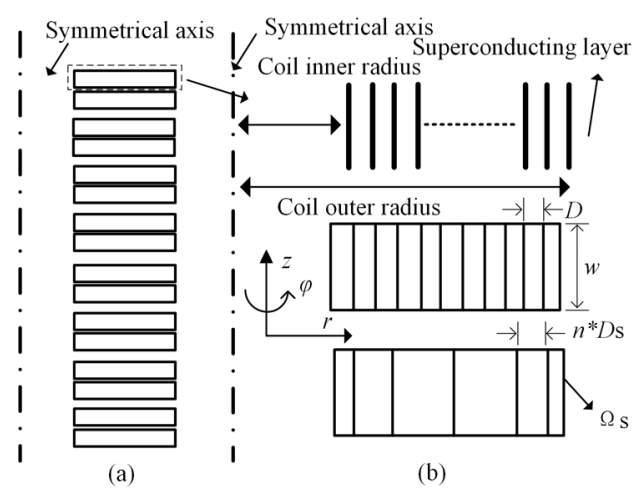

Figure 1. Basic structure and homogenization method.

TABLE I. THE PARAMETERS OF HTS AIR-CORE TRANSFORMER

\begin{tabular}{|c|c|}
\hline Parameters & Value \\
\hline Inner diameter $(\mathrm{cm})$ & 12 \\
\hline Outer diameter $(\mathrm{cm})$ & 13.2 \\
Air gap $(\mathrm{cm})$ & 1 \\
\hline Number of double-pancake & 8 \\
\hline Turn number & $2 \times 20$ \\
\hline Tape width $(\mathrm{mm})$ /thickness $(\mathrm{mm})$ & $4.5 / 0.3$ \\
\hline Superconducting layer thickness $(\mathrm{um})$ & 1 \\
\hline Critical current $I_{\mathrm{c}}$ at $77 \mathrm{~K}(\mathrm{~A})$ & 143.96 \\
\hline
\end{tabular}

Here the $J_{\mathrm{c}}(B)$ is dependent on the perpendicular and parallel magnetic field, where $B_{0}=35 \mathrm{mT}, J_{c 0}=7.545 \times$ $10^{8} \mathrm{~A} / \mathrm{m}^{2}, \alpha=0.6, \beta=0.25$. The AC loss per cycle is calculated as follows:

$$
Q=\sum 2 \int_{T / 2}^{T} d t\left(\int_{\Omega_{\mathrm{s}}} E \cdot J d \Omega_{s}\right)
$$

\section{B. Homogenization Method}

The homogenization method is briefly shown in Fig. 1(b). First, the superconducting layer is artificially expanded to make tapes fill the space of the entire pancake. Then combine several adjacent turns of a pancake to a new turn because adjacent tapes have similar current density distributions. Note that a new current constraint should be used to ensure the transport current in the new turn is still the same as the original mode. 




(a)

Figure 2. (a) Magnetic field (b) normalized current density (c)AC loss.

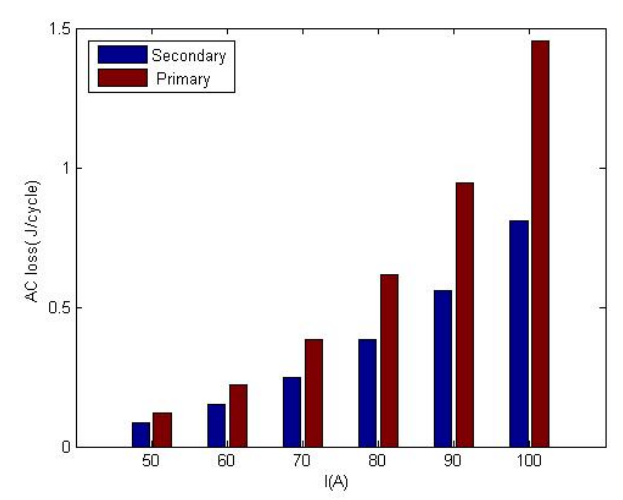

Figure 3. AC loss per cycle when different primary current levels.

\section{RESULTS AND DISCUSSION}

\section{A. Electromagnetic Field and AC loss Analysis}

When a current of $60 \mathrm{~A} / 50 \mathrm{~Hz}$ passes through the primary side, the electromagnetic field distribution of the double pancake coils at the current peak are shown in the Fig. 2. Coupling occurs between the primary and secondary coils, and an induced current appears on the secondary coils which confirms the correctness of the model. The instantaneous AC loss power of the primary and secondary coils is shown in Fig. 2 (c). Fig. 3 shows the AC loss of the primary and secondary coil at different current level.

\section{B. Validation of Effect}

Assume the load impedance is $10 \Omega$, the system efficiency can be obtained from equation (6). The cryocooler power is calculated as 12 times of the AC loss power of the superconductor at $77 \mathrm{~K}$ [3]. The system efficiency including cryocooler power loss is defined as equation (7). When different currents are applied on the primary side, the system efficiency $\eta$ and system efficiency $\eta_{\mathrm{c}}$ including cryocooler are shown in Fig. 4.

$$
\begin{gathered}
\eta=P_{\text {out }} /\left(P_{\text {out }}+P_{\text {hys }}\right) \\
\eta_{\mathrm{c}}=P_{\text {out }} /\left(P_{\text {out }}+P_{\text {hys }}+P_{c y}\right)
\end{gathered}
$$

\section{Air gap optimization analysis}

As the air gap changes, the system efficiency changes are shown in Fig. 5. When the air gap of the system is reduced,

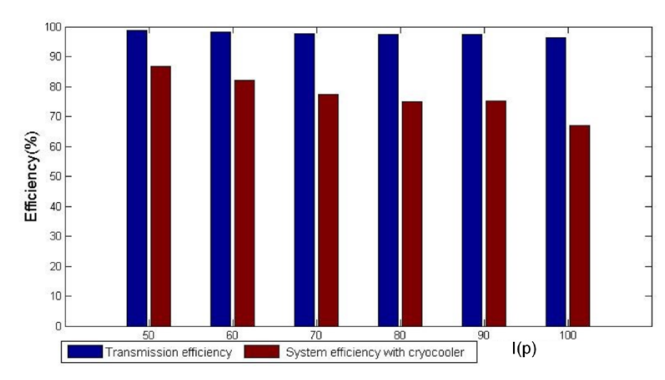

Figure 4. Transmission efficiency varies with current.

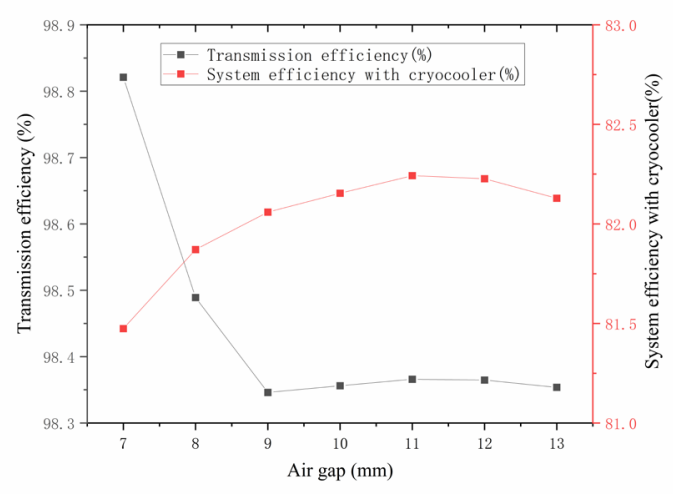

Figure 5. Transmission efficiency varies with air gap.

the induced current of the secondary coil increases and the transmission efficiency of the system increases. However, increasing the current will increase both the AC loss of the system and cryocooler power loss, leading to a decrease in efficiency. Therefore, this system can increase the $1 \mathrm{~mm}$ air gap, so that the system efficiency including the cryocooler power loss increases and the transmission efficiency does not change much.

\section{CONCLUSIONS}

In this paper, the HTS air-core transformer model is established based on the $\boldsymbol{H}$-formulation. The magnetic field distribution and critical current distribution of primary and secondary coils are obtained. The AC loss of the primary and secondary coils and the efficiency of the system when different currents pass through are analyzed. It is found that increasing the air gap by $1 \mathrm{~mm}$ would increase the system efficiency including the cryocooler power loss increases and the transmission efficiency does not change much. The analysis method is provided to optimize the design of HTS air-core transformer.

\section{REFERENCES}

[1] J. X. Jin, Y. J. Tang, X. Y. Xiao, B. X. Du, Q. L. Wang, J. H. Wang, S. H. Wang, Y. F. Bi and J. G. Zhu, "HTS power devices and systems: principles, characteristics, performance, and efficiency," IEEE Trans. on Appl. Supercond., vol. 26, no. 7, pp. 3800526.

[2] B. Shen, F. Grilli, and T. Coombs, "Review of the AC loss computation for HTS using H formulation," Superconductor Science and Technology, vol. 33, no. 3, p. 033002.

[3] R. Inoue, K. Igarashi, Y. Nagasaki, D. Miyagi, M. Tsuda, and H. Matsuki, "Electric Power Transmission Characteristics of a Wireless Power Transmission System Using High Temperature Superconducting Coils for Railway Vehicle," IEEE Transactions on Applied Superconductivity, vol. 29, no. 5, pp. 1-5. 